\title{
Broadband Multifunctional Efficient Meta-Gratings Based on Dielectric Waveguide Phase Shifters
}

\section{Citation}

Khorasaninejad, Mohammadreza, and Federico Capasso. 2015. "Broadband Multifunctional Efficient Meta-Gratings Based on Dielectric Waveguide Phase Shifters." Nano Letters 15 (10): 6709-15. https://doi.org/10.1021/acs.nanolett.5b02524.

\section{Permanent link}

http://nrs.harvard.edu/urn-3:HUL.InstRepos:41371318

\section{Terms of Use}

This article was downloaded from Harvard University's DASH repository, WARNING: This file should NOT have been available for downloading from Harvard University's DASH repository.

\section{Share Your Story}

The Harvard community has made this article openly available.

Please share how this access benefits you. Submit a story.

\section{Accessibility}




\title{
Broadband Multifunctional Efficient Meta-Gratings Based on Dielectric Waveguide Phase Shifters
}

\author{
Mohammadreza Khorasaninejad* and Federico Capasso* \\ School of Engineering and Applied Sciences, Harvard University, Cambridge, Massachusetts 02138, United States
}

\section{Supporting Information}

ABSTRACT: Molding the wavefront of light is a basic principle of any optical design. In conventional optical components such as lenses and waveplates, the wavefront is controlled via propagation phases in a medium much thicker than the wavelength. Metasurfaces instead typically produce the required phase changes using subwavelength-sized resonators as phase shift elements patterned across a surface. This "flat optics" approach promises miniaturization and improved performance. Here we introduce metasurfaces which use dielectric ridge waveguides (DRWs) as phase shift elements in which the required phase

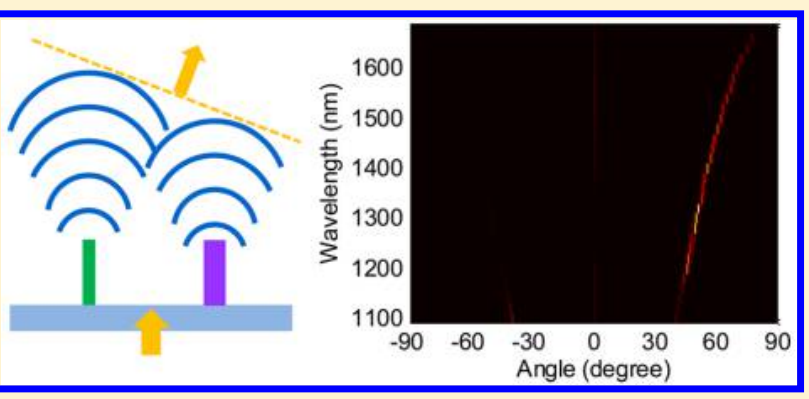
accumulation is achieved via propagation over a subwavelength distance. By engineering the dispersive response of DRWs, we experimentally realize high resolving power meta-gratings with broadband $(\lambda=1.2-1.7 \mu \mathrm{m})$ and efficient routing (splitting and bending) into a single diffraction order, thus overcoming the limits of blazed gratings. In addition, we demonstrate polarization beam splitting capabilities with large suppression ratios.

KEYWORDS: Metasurfaces, meta-grating, dielectric waveguide, subwavelength structure, dispersion engineering

$\mathrm{M}$ etamaterials open new opportunities in optics by overcoming the limits set by natural material properties. ${ }^{1,2}$ Phenomena such as perfect lensing, ${ }^{3}$ optical cloaking, ${ }^{4}$ and negative index of refraction ${ }^{5}$ are some examples of these novel properties. In recent years, metasurfaces have emerged and added optical functionalities with the advantage of simple fabrication compared to 3D-metamaterials. ${ }^{6,7}$ A metasurface consists of an array of subwavelength-spaced phase shift elements laid out in a pattern on a flat surface to control the wavefront of light. Based on this approach, various optical functionalities have been demonstrated in very compact and flat configurations including beam shaping, ${ }^{8,9}$ lensing, ${ }^{10-14}$ beam bending, ${ }^{14,15}$ and polarization splitting. ${ }^{15-20}$ The phase shift elements of a metasurface typically satisfy two criteria, namely, they (i) have subwavelength size and spacing and (ii) create a phase shift in the whole $2 \pi$ range to achieve full control of the wavefront. These criteria are met by metallic and dielectric resonators. 6,7

In this work, we experimentally demonstrate a new type of subwavelength-sized phase shifter based on dielectric ridge waveguides (DRWs) with the ability to efficiently route (split/ bend) the light over a broad range of wavelengths in the nearinfrared (NIR). In addition, our approach does not face issues of high absorption losses, polarization conversion, scattering efficiency and fabrication complexity, which are unavoidable for various metasurface designs. $6,7,21-23$

A ubiquitous functionality in free space and integrated optics is wavelength demultiplexing with applications ranging from spectroscopy to communication. ${ }^{24}$ This requires the development of blazed diffraction gratings in which the majority of transmitted or reflected light is funneled into a single order. Generally, blazed gratings are designed so that the incident beam experiences a gradual $2 \pi$ phase shift over a grating period, which is achieved through either a continuous profile or a staircase approximation. The former can be implemented by triangular grooves, ${ }^{24}$ and the latter is achieved using gradedindex material available by recent progress of nanofabrication techniques. ${ }^{25-28}$ Regardless of the grating configuration, chromatic dispersion of the material/elements of the blazed grating makes it very challenging to maintain high efficiency over a broad range of wavelengths. ${ }^{24-27,29}$ In other words, by operating at a wavelength away from the design wavelength, the light will be diffracted to undesired orders, thus reducing the grating efficiency. Ribot et $\mathrm{al}^{28}$ used a combination of pillars and holes as artificial phase elements to create a relatively achromatic phase variation over the period of the grating. An absolute grating efficiency of $\sim 50 \%$ was achieved over a broad range of wavelengths around $10 \mu \mathrm{m}$. A grating period of $\Lambda=$ $140 \mu \mathrm{m}$ and a center wavelength of $\lambda \approx 10 \mu \mathrm{m}$ resulted in a deflection angle of $\sim 4^{\circ}\left(\theta=\sin ^{-1}(\lambda / \Lambda)\right)$. The small deflection angle deteriorates the resolving power of the grating and limits its ability to separate nearby wavelengths. However, keeping the grating blazed is challenging particularly when the grating operates at a large deflection angle. This is a long-standing obstacle, called the shadow effect, to obtain highly efficient blazed gratings. ${ }^{30}$ The shadow effect significantly degrades the

Received: June 25, 2015

Revised: September 11, 2015 

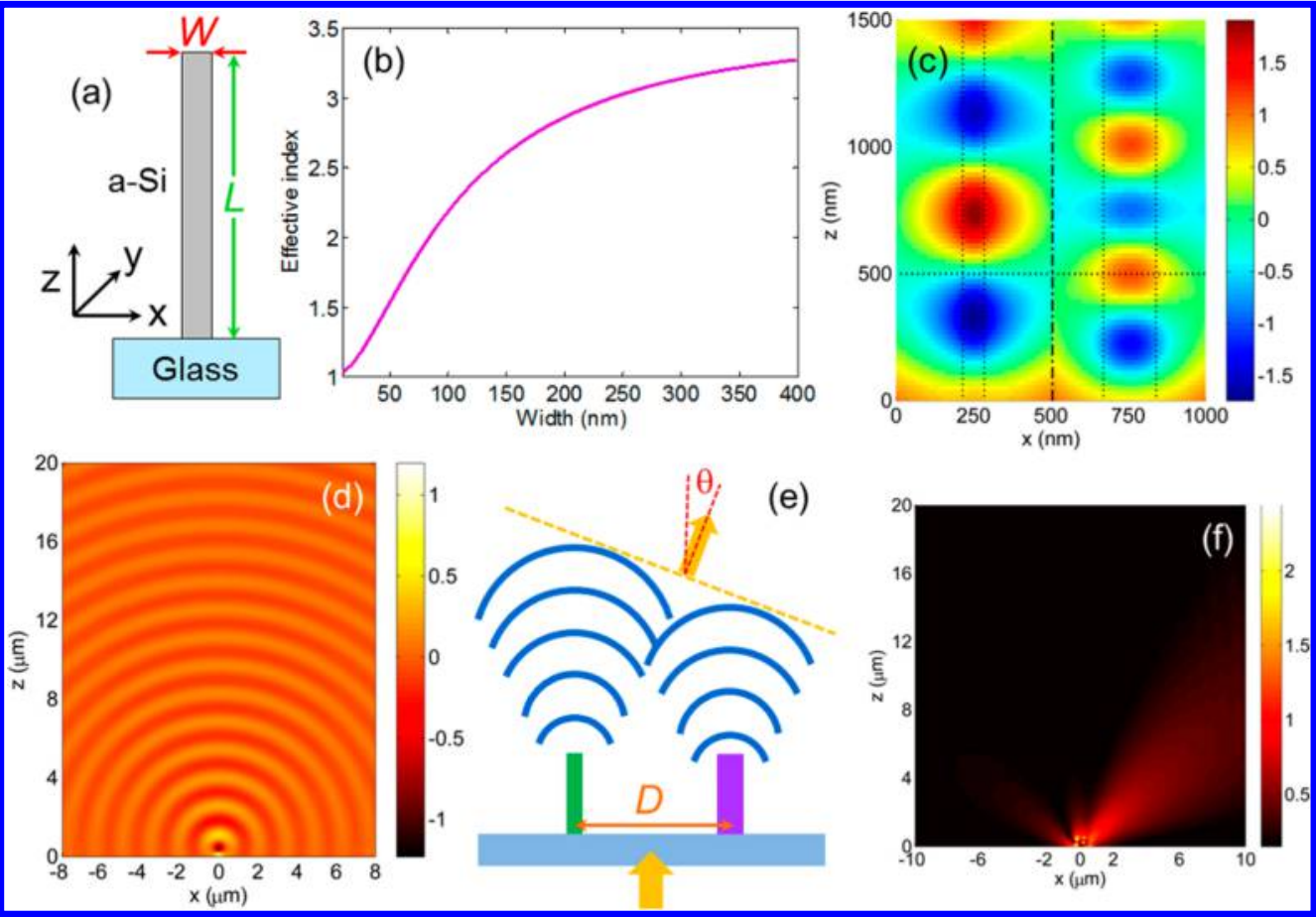

Figure 1. (a) Schematic diagram of dielectric ridge waveguide (DRW), an amorphous-silicon nanoridge on a glass substrate. The DRW has a deepsubwavelength width $(W)$ and strongly confines the light along $x$. In the simulation the DRW is infinitely extended along $y$. In the fabricated structures the DRWs are at least $125 \mu \mathrm{m}$-wide along $y$. (b) Effective index of DRW as a function of its width for parallel polarization $\left(E_{y}\right)$. (c) Electric field (real $\left[E_{y}\right]$ ) distribution in $x z$-plane showing the emergence of a phase difference between two DRWs with different widths along a subwavelength propagation distance. For instance, a phase difference $\Delta \varphi \approx 100(0.55 \pi)$ is obtained after a propagation length $L=500 \mathrm{~nm} \approx \lambda / 3$, marked by a horizontal dotted line. Because of strong confinement in the DRWs, waveguide coupling is negligible. Left and right DRWs have widths of $70 \mathrm{~nm}$ and $170 \mathrm{~nm}$, respectively. Boundaries of the DRWs are highlighted by vertical dotted lines. Simulations are performed for single DRW under plane-wave excitation and parallel polarization (electric field along the $y$ ) at $\lambda=1425 \mathrm{~nm}$. Perfectly matched layer and periodic boundary conditions are used for $z$ and $x$, respectively. (d) Electric field (real $\left[E_{y}\right]$ ) distribution in $x z$-plane. The DRW radiates into free space. It is located at $x$ $=0$ and has width $W=70 \mathrm{~nm}$ and length $L=500 \mathrm{~nm}$. (e) Two DRWs of different widths placed at a subwavelength distance $D$ radiate in free space with a phase difference. These radiated beams constructively interfere at the angle $\theta$ creating a wavefront in that direction. (f) Simulation of light bending by two DRWs with widths of $70 \mathrm{~nm}$ (located on left) and $170 \mathrm{~nm}$ (located on the right) and center-to-center distance $D=450 \mathrm{~nm}(\sim \lambda / 3)$. For this simulation, two DRWs are located at the center of a $1.7 \mu \mathrm{m}$-wide aperture and the wavelength is $1425 \mathrm{~nm}$.

performance of the grating when the period is in the order of a few wavelengths or less. ${ }^{30}$ A smaller period demands a higher phase gradient, which is harder to preserve over a broad range of wavelengths. Here, we overcome the above limits by a conceptually new design where we engineer the dispersive response of phase shifting waveguide elements.

Figure 1a shows our proposed DRW, phase shift element, made of amorphous silicon (a-Si). The DRW acts as a slab waveguide with the propagation direction along the $+z$. Figure $1 \mathrm{~b}$ shows that by adjusting the DRW width, the effective index of its fundamental mode can vary anywhere from $n_{\text {eff }} \approx 1$ (when the light is mostly in air) to $n_{\mathrm{eff}} \approx n_{\mathrm{a}-\mathrm{Si}}$ (when the light is mostly in $\mathrm{a}-\mathrm{Si}$ ). If we have two DRWs side-by-side, with different widths and negligible optical coupling between the DRWs, then light traveling down the different DRWs will accumulate a phase shift $\Delta \varphi$ proportional to their length $L$ :

$$
\Delta \varphi=\frac{2 \pi}{\lambda} \Delta n_{\mathrm{eff}} L
$$

where $\Delta n_{\text {eff }}$ is the effective index difference between the two DRWs. A phase difference of $\Delta \varphi=2 \pi$ can be obtained over a subwavelength propagation length of:

$$
L=\frac{\lambda}{\Delta n_{\mathrm{eff}}}
$$

if the two DRWs are designed with a $\Delta n_{\text {eff }}$ that exceeds unity. Achieving $\Delta \varphi=2 \pi$ is pivotal to have full control of the wavefront.

Figure 1c depicts the emergence of the phase difference between two waveguides of different widths. For these simulations, we consider semi-infinitely long DRWs and set perfectly matched layer (PML) at the $z$-boundaries, so that end-facet reflections are eliminated. This assumption helps visualize mode propagation and the appearance of the phase difference between two optical modes; and despite neglecting end-facet reflections, our model is quantitatively predictive as discussed next.

Owing to its high index-contrast, the a-Si waveguide can confine light in a subwavelength region. Figure S1a,b of Supporting Information (SI) shows the optical modes of 70 and $170 \mathrm{~nm}$-wide slab waveguides at a wavelength of $1425 \mathrm{~nm}$. The full width at half maximums (fwhm) of the modes' field profiles of both DRWs are $\sim 240 \mathrm{~nm}$, confirming deep subwavelength (fwhm $\approx \lambda / 6)$ confinement of the optical modes. Consequently, when the beam reaches the DRW's endfacet, it couples out into free space uniformly in the $x z$-plane as shown in Figure 1d. This is expected from the multipole expansion: A radiator much smaller than a wavelength tends to emit approximately a dipole radiation pattern. It is notable that due to high mode confinement (fwhm of $\sim 240 \mathrm{~nm}$ ) and large 


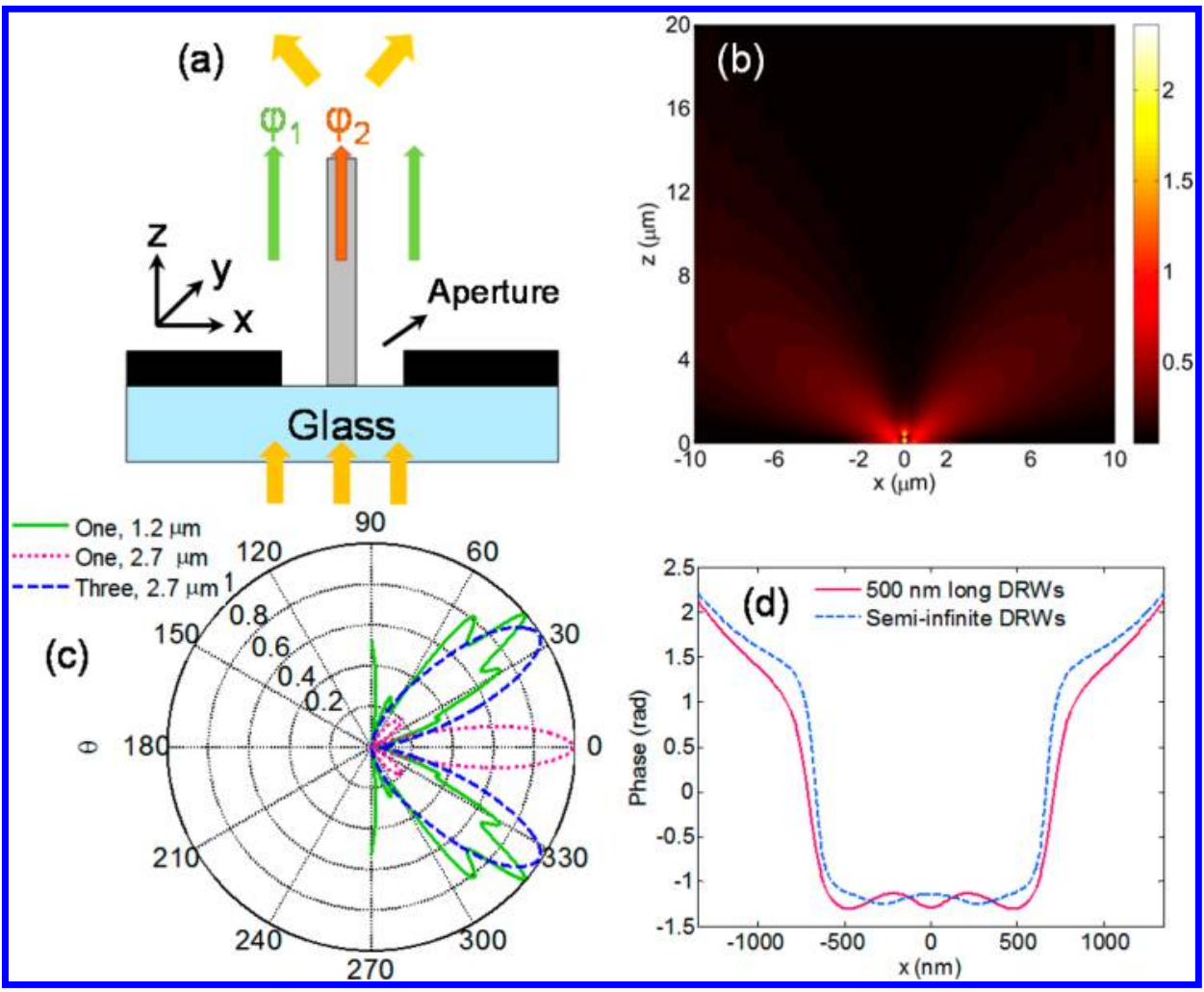

Figure 2. (a) Schematic diagram illustrating the mechanism of light-splitting by dielectric ridge waveguide (DRW). Destructive interference in the forward direction occurs when the phase difference, $\Delta \varphi=\varphi_{2}-\varphi_{1}$, between the light paths in the DRW and in the air, indicated by orange and green arrows, respectively, is equal to $\Delta \varphi=\pi$. (b) Simulation of light-splitting using a single DRW (width $W=100 \mathrm{~nm}$, length $L=500 \mathrm{~nm}$ ). Simulation is performed at $\lambda=1450 \mathrm{~nm}$ and for parallel polarization (electric field along the $y$ ). The DRW is placed at the center of a $1.2 \mu \mathrm{m}$-wide aperture in an opaque screen. (c) Normalized far-field response (real $\left.\left(E_{y}\right)^{2}\right)$ of DRWs all of the same width $(100 \mathrm{~nm})$ and length $(500 \mathrm{~nm})$ : (i) single DRW placed in the middle of a $1.2 \mu \mathrm{m}$-wide aperture; (ii) single DRW in a $2.7 \mu \mathrm{m}$-wide aperture. Appearance of the unwanted lobe at $\theta=0$ is due to the fact that scattering is dominated by diffraction from the large aperture; (iii) three identical DRWs separated by $500 \mathrm{~nm}$ in a $2.7-\mu \mathrm{m}$ wide aperture. It is apparent that the majority of transmitted light is split. (d) Phase distribution recorded at a distance of 10 nm above the facets of the DRWs (case (iii)). We repeated the simulation with semi-infinitely long DRWs to exclude the Fabry-Perot effects and found practically identical phase distribution. In all the simulations, the incident light is polarized along $y$.

index difference together with the short propagation distance $(500 \mathrm{~nm} \approx \lambda / 3)$ waveguide coupling is negligible (Figure S1ce of SI).

While 70 and $170 \mathrm{~nm}$-wide DRWs can have a distinctive phase difference (Figure 1b,c), they generate similar free space propagation patterns (see Figure S2 of SI). This is expected due to the similarity with nearly equal fwhm of the DRWs optical modes. The ability to control DRWs' propagation phase (by adjusting the DRWs' width) without corresponding changes in their radiation patterns make them ideal metasurface phase shift elements. In addition, the strong confinement of their optical modes enables the DRWs to be packed very densely, with subwavelength separation and minimal coupling.

We start by considering the simple case of just two phase shift elements (DRWs) within an aperture. By placing two DRWs with phase difference of $\Delta \varphi$ at a subwavelength distance $D$, one can direct the beam to an angle $\theta$ as illustrated in Figure 1e. The value of $\theta$ depends on $\Delta \varphi, \lambda$, and $D$, and is governed by the condition of constructive interference along the direction defined by $\theta$ :

$$
\Delta \varphi=\frac{2 \pi D}{\lambda} \sin (\theta)
$$

Now consider two DRWs with the same length $L=500 \mathrm{~nm}$, and different widths (70 and $170 \mathrm{~nm}$ ), placed at a center-tocenter distance $D=450 \mathrm{~nm}$. These DRWs have the phase difference $\Delta \varphi=95^{\circ}(0.52 \pi)$ at wavelength $\lambda=1425 \mathrm{~nm}$. This phase difference is very close to $\Delta \varphi=100^{\circ}$, that of semiinfinitely long DRWs (see Figure 1c). Based on eq 3 the beam should be redirected by the DRWs to $\theta=55^{\circ}$, which is also obtained by the finite difference time domain (FDTD) simulation shown in Figures If and S3 of SI. We note the importance of the DRWs' subwavelength distance (here, $D=$ $\lambda / 3)$ to avoid multiple diffraction orders. This bypasses the fundamental limitation of conventional diffractive elements in which the presence of spurious diffraction orders is unavoidable. For instance the latter accounts for the origin of multiple real and virtual focal points in Fresnel zone plates. ${ }^{31}$

A similar approach can be adapted to split light rather than to bend it. This light-splitting is conceptually illustrated in Figure $2 \mathrm{a}$ where the DRW is located in the middle of an aperture in an opaque screen. The required phase difference is achieved through light propagating in the $100 \mathrm{~nm}$-wide DRW (orange arrow in Figure 2a) and air adjacent to it (green arrow in Figure 2a). We choose the length of the DRW to create a $\Delta \varphi=\pi$ phase difference compared to propagation in air, based on its waveguide effective index as described above. This ensures destructive interference in the forward direction shown in Figure $2 b$. Figure $2 \mathrm{c}$ shows that a single DWR has a limited scattering cross section. For a $1.2 \mu \mathrm{m}$-wide aperture and an individual $100 \mathrm{~nm}$-wide DRW the majority of the incident light is split (Figure 2c). By expanding the aperture width to $2.7 \mu \mathrm{m}$, 

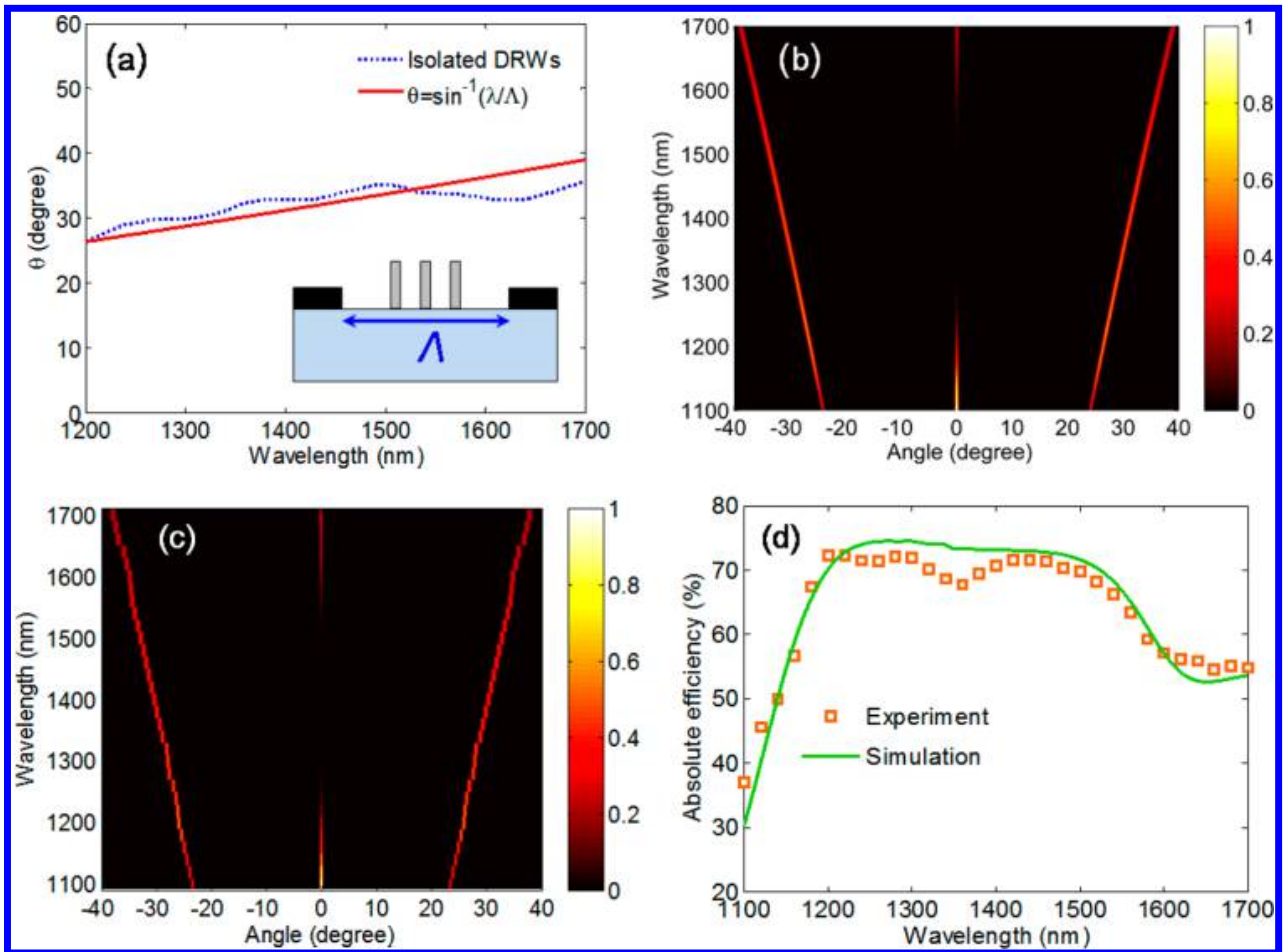

Figure 3. (a) Dielectric ridge waveguides (DRWs) and meta-grating are designed so that the dispersion of the far-field response of the former matches that of the latter. Shown in dotted line is deflection angle of three isolated DRWs (see inset) with center-to-center distance $500 \mathrm{~nm}$ and identical lengths and widths $(L=500 \mathrm{~nm}$ and $W=105 \mathrm{~nm})$ for normally incident light polarized along $y$ (Figure 2a). The deflection angle is calculated from the far-field response of DRWs using finite difference time domain simulations. The deflection angle for normal incidence of the meta-grating is calculated from $\theta=\sin ^{-1}(\lambda / \Lambda, \Lambda=2700 \mathrm{~nm})$ in which $\Lambda$ is the period. (b,c) Simulated (b) and measured (c) far-field response (power) of meta-grating with three DRWs as its phase shift elements. It is evident that for a broad range of wavelengths (from 1200 to $1700 \mathrm{~nm}$ ) the majority of the transmitted light is directed to the first order of the meta-grating. Fabricated DRWs have $\mathrm{L}=500 \mathrm{~nm}, \mathrm{~W}=105 \mathrm{~nm}$, and center-tocenter distance of $500 \mathrm{~nm}$. The overall extent of the meta-grating is $125 \mu \mathrm{m} \times 125 \mu \mathrm{m}$. (d) Measured and simulated absolute efficiency (ratio of transmitted power, which is funneled to first orders to the input power) of the meta-grating versus wavelength.

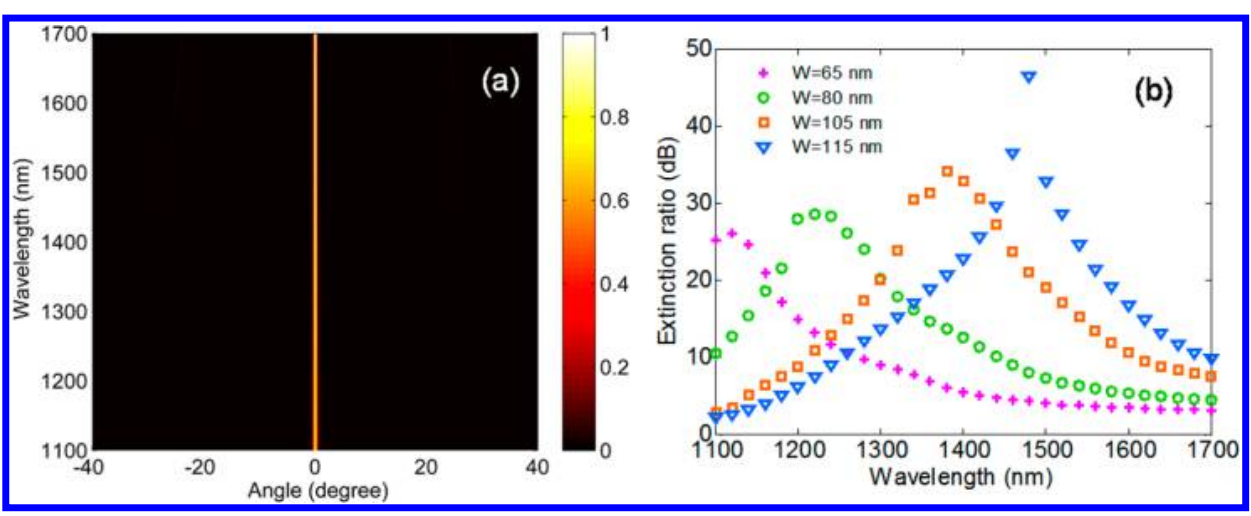

Figure 4. Meta-grating also acts as polarization beam splitter (PBS) allowing a beam polarized along $x$ (see Figure 2a) to pass through undisturbed while splitting light polarized along $y$. (a) Simulated far-field response (real $\left.\left(E_{y}\right)^{2}\right)$ of meta-grating for perpendicular polarization (electric field is along $x$ ). The meta-grating allows this polarization to pass through with minimal deflection. (b) Measured extinction ratios for several different designs. The center wavelength of the PBS can be tuned by adjusting the DRWs width in the $65-115 \mathrm{~nm}$ range without changing other parameters. For all of these designs, three dielectric ridge waveguides are used with center-to-center distance of $500 \mathrm{~nm}$, length $L=500 \mathrm{~nm}$, and period $\Lambda=2700$ $\mathrm{nm}$.

the scattering cross section dramatically decreases and the farfield is dominated by diffraction from the aperture. Note that the large forward scattering $\left(\theta=0^{\circ}\right)$ implies a negligible scattering cross section of the DRW. To increase the cross section, we increase the number of DRWs to three. The farfield response of this case is also shown in Figure $2 \mathrm{c}$ where three $100 \mathrm{~nm}$-wide DRWs increase the cross section approximately to the full aperture width and split almost $100 \%$ of the transmitted light.

We also simulated the phase shift induced by DRWs as shown in Figure $2 \mathrm{~d}$. The phase is recorded at a distance of 10 $\mathrm{nm}$ above the tip of DRWs. A phase difference (between air and DRWs) of $\sim \pi$ is achieved, which results in destructive interference at $\theta=0^{\circ}$. Based on eq 3 , the constructive interference should occur at an angle $\theta=32^{\circ}$, which is close to 
the value $\theta=33^{\circ}$ predicted by far-field calculation (Figure $2 \mathrm{c}$ ). We repeated the simulation in which the DRWs are semiinfinitely long to eliminate any Fabry-Perot effects and recorded the phase at the same place. As shown in Figure 2d, the phase distribution is very similar to that of the previous case confirming that the phase accumulation of DRWs is due to the waveguiding effect. Although the reflection at the facet is inevitable because of index-mismatching between the DRW and air, this reflection does not have a significant effect on the phase.

As shown in Figure S4 of SI, our meta-grating of period $\Lambda$ consists of unit cell of the same size comprising three DRWs. For broadband operation, the DRWs are designed to diffract normally incident light into the first order of the grating for all wavelengths. Our simulation shows that this broadband blazed grating condition can be achieved with very good approximation design of Figure 3a. The corresponding designed effective index of three isolated DRWs is shown in Figure S5 of SI. Figure $3 b, c$ theoretically and experimentally confirms that our design is effective in achieving a highly efficient metagrating for a broad range of wavelengths. The measured absolute efficiency of meta-grating is illustrated in Figure $3 \mathrm{~d}$ showing high efficiency at most wavelengths and good agreement with simulations.

This meta-grating behaves quite differently for polarization perpendicular to the ridge axis as compared to parallel polarization. As shown in Figure $4 \mathrm{a}$ for this polarization more than $95 \%$ of light is transmitted undisturbed for wavelengths ranging from $1100 \mathrm{~nm}$ to $1700 \mathrm{~nm}$. These results are also consistent with calculations shown in Figure S1 of SI in which the effective index of DRW for widths of interest $(W<150$ $\mathrm{nm}$ ) is close to one (air) for the perpendicular polarization. In other words, the meta-grating acts as a broadband polarization beam splitter (PBS). This polarization management is more efficient than that of conventional wire grid polarizers in which the undesired polarization is discarded via absorption or reflection process. Therefore, the overall efficiency of the PBS (more than $95 \%$ and $70 \%$ for perpendicular and parallel polarization, respectively) is well beyond the theoretical limits of $50 \%$ for wire grid polarizers. In addition, the meta-gratings with ultrathin and compact architectures facilitate their integration into compact systems which otherwise would not be possible with conventional bulky PBS. The extinction ratio of the PBS is measured and illustrated in Figure $4 \mathrm{~b}$. The high extinction ratio (more than $45 \mathrm{~dB}$ ) and efficiency of this PBS is potentially important for various applications such as polarization resolved imaging and microscopy.

Highly efficient light bending also can be achieved by modifying the design to suppress the -1 -order and funnel the light to the +1 -order. Figure 5a shows the scanning electron micrograph (SEM) of the fabricated meta-grating consisting of two DRWs with 50 and $150 \mathrm{~nm}$ widths, $L=500 \mathrm{~nm}$, and $D=$ $450 \mathrm{~nm}$ (Figure $5 \mathrm{~b}$ ). The DRW meta-grating period is $\Lambda=$ $1700 \mathrm{~nm}$. The main aim of this design is to increase the resolving power of the meta-grating by increasing diffraction angle (Figure S6 of SI). As shown in Figure 5c, absolute efficiency of more than $80 \%$ is achieved at wavelength $\lambda=1300$ nm with a large deflection angle $\theta=50^{\circ}\left(\sin ^{-1}(1300 / 1700)=\right.$ $\left.50^{\circ}\right)$ ). Moreover, highly efficient light-bending (50\%) is maintained at a very large deflection angle $\theta=65^{\circ}$ (wavelength $\lambda=1550 \mathrm{~nm}$ ). Similar to the previous case (splitting), this meta-grating also allows the majority of the perpendicularly polarized beam to pass through undisturbed thereby behaving

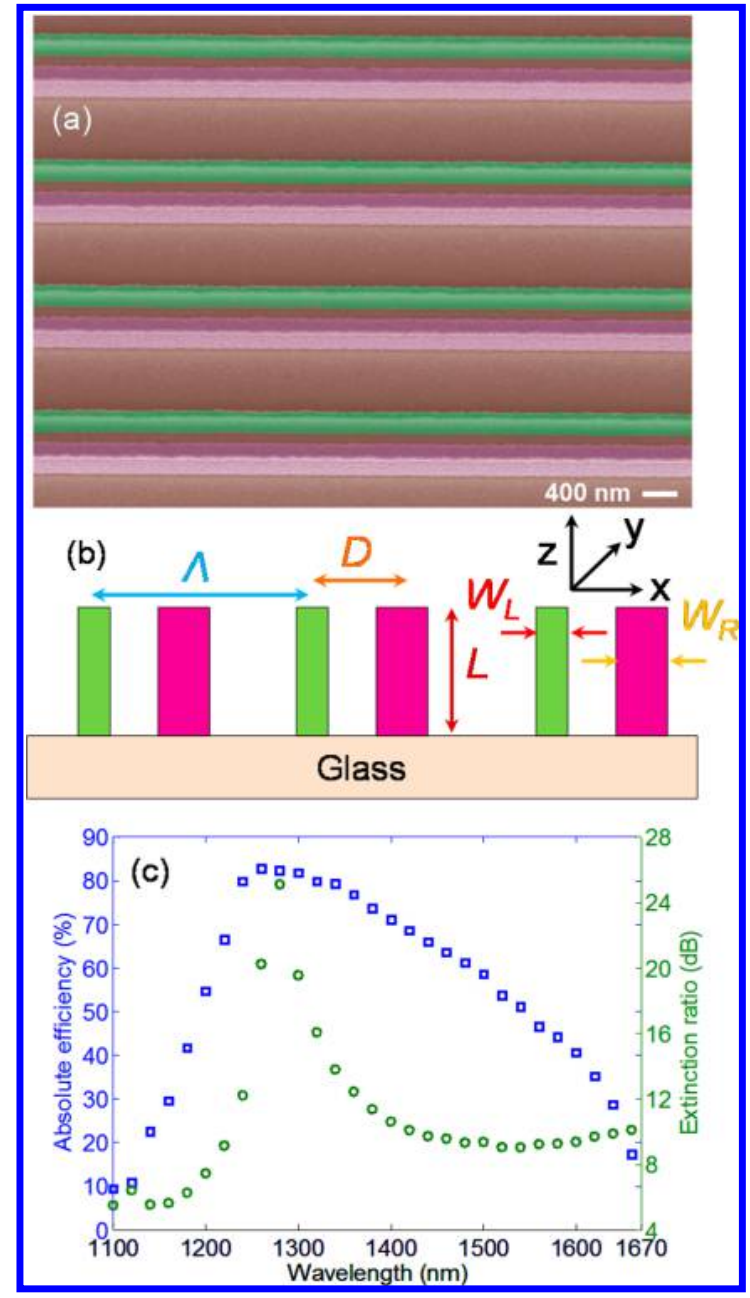

Figure 5. Meta-grating for efficient and broadband light bending. (a) False-colored side-view SEM of meta-grating. (b) Schematic diagram of meta-grating showing the dimensions of meta-grating's elements, dielectric ridge waveguides (DRWs), and period. Meta-grating elements are two DRWs with different widths $W_{\mathrm{L}}$ (left waveguide) and $W_{\mathrm{R}}$ (right waveguide). DRWs have $W_{\mathrm{L}}=50 \mathrm{~nm}$ and $W_{\mathrm{R}}=150$ $\mathrm{nm}, D=450 \mathrm{~nm}$, and $L=500 \mathrm{~nm}$. The period of meta-grating is $\Lambda=$ $1700 \mathrm{~nm}$. (c) Experimental results for absolute efficiency (ratio of transmitted power, which is funneled into the +1 -order to the input power) of the meta-grating as a function of wavelength. The measured extinction ratio of the meta-grating is also shown when used as polarization beam splitter.

as a PBS. The extinction ratio of the PBS is measured and shown in Figure 5c.

In summary, we have presented a new concept for wavefront shaping using DRWs. The DRWs' capability to control the phase via subwavelength propagation with minimal loss highlights their promise as building blocks to realize versatile optical functionalities in an ultracompact and efficient configuration. We experimentally demonstrated meta-gratings with broadband and efficient routing (splitting and bending) into a single diffraction order and additional PBS capabilities. Note that broadband and efficient operation is not possible with either conventional blazed gratings ${ }^{25,26,32}$ or light bending components based on metamaterials and metasurfaces. $8,13,14$ Our approach has also the attractive features of straightforward fabrication, design flexibility, and reproducibility on large scales. When integrated with an image sensor, the proposed metagrating could enable high efficiency spectroscopy and imaging. 
In addition, there is the obvious potential for extending the concept to more spectral bands, especially into the $\mathrm{mid} /$ farinfrared where a-Si maintains its low loss.

It is interesting to relate this work to the extensive research carried out by the group of Chang-Hasnain ${ }^{34}$ on subwavelength dielectric structures. ${ }^{35}$ In that work the main goal was to achieve high reflectivity broadband mirrors; this was accomplished by suppression of forward scattering via destructive interference between the two grating modes.

Methods. DRWs Fabrication. First, the substrate (fused silica) is cleaned via acetone sonication followed by an oxygen plasma. Plasma-enhanced chemical vapor deposition (PECVD) is then used to deposit $500 \mathrm{~nm}$ a-Si. The sample is spin-coated with poly(methyl methacrylate) (PMMA, $950 \mathrm{~K} \mathrm{~A} 2$ ) at a speed of $6000 \mathrm{rpm}$ and baked on a hot plate at $180{ }^{\circ} \mathrm{C}$ for $5 \mathrm{~min}$. Next, $5 \mathrm{~nm}$ chromium ( $\mathrm{Cr}$ ) is evaporated on PMMA to avoid charging effects in the electron beam lithography (EBL) step because of poor conductivity of the glass substrate $(\mathrm{Cr}$ is removed right after EBL). EBL is consequently carried out by Elionix ELS-F125 at $125 \mathrm{kV}$. The exposed resist is then developed in a mixture of methyl isobutyl ketone and isopropyl alcohol (MIBK/IPA 1:3) for $60 \mathrm{~s}$, dipped in IPA for $30 \mathrm{~s}$, and blown dry using nitrogen. Aluminum ( $\mathrm{Al}, 20 \mathrm{~nm}$ ) is then thermally deposited. Thus, the lift-off process is accomplished by soaking the sample overnight in a solvent stripper (Remover PG from Microchem). Inductively coupled plasma-reactive ion etching (ICP-RIE) is performed to etch the DRWs. ${ }^{33}$ The $\mathrm{Al}$ mask is removed by soaking in developer (MF319 from Shipley) for $5 \mathrm{~min}$. The overall dimension of the fabricated meta-gratings is $125 \mu \mathrm{m} \times 125 \mu \mathrm{m}$ for splitting and $600 \mu \mathrm{m} \times$ $600 \mu \mathrm{m}$ bending case. As the incident beam size $(4 \mathrm{~mm} \times 4$ $\mathrm{mm}$ ) used for measurement is larger than the size of metagrating, the area around meta-grating is blocked by depositing $150 \mathrm{~nm}$ thick $\mathrm{Al}$. The latter is done through optical lithography and lift-off process.

Measurements and Simulations. The measurement setup includes a supercontinuum laser (NKT "SuperK") equipped with a set of acousto-optic tunable filters (NKT "Select") with a fiber output. The fiber is connected to a fiber coupled collimator creating a collimated beam with size of $4 \mathrm{~mm} \times 4$ $\mathrm{mm}$. The collimated beam then passes through a linear polarizer, which is adjusted appropriately for each measurement. Light transmitted through the meta-grating is measured by an InGaAs photodetector (Thorlabs DET10D) mounted on a rotation stage. All measurements are normalized to the power passing through an aperture (with the same size of metagrating) fabricated on a glass substrate

All simulations are performed in $2 \mathrm{D}$ due to the large extension of the meta-gratings using the FDTD method (FDTD solution package from Lumerical Inc.). For metagrating simulations, we apply periodic boundary conditions at the $x$ - and PML at $z$-boundaries. For isolated DRWs cases (non- periodic DRWs) we assume PML conditions for both $x$ and $z$-boundaries.

\section{ASSOCIATED CONTENT}

\section{S Supporting Information}

The Supporting Information is available free of charge on the ACS Publications website at DOI: 10.1021/acs.nanolett.5b02524.

Design details and supplementary figures (PDF)

\section{AUTHOR INFORMATION}

\section{Corresponding Authors}

*E-mail: capasso@seas.harvard.edu. Phone: 617384 7611. Fax: 6174952875.

*E-mail: khorasani@seas.harvard.edu.

\section{Notes}

The authors declare no competing financial interest.

\section{ACKNOWLEDGMENTS}

This work was supported in part by the Air Force Office of Scientific Research (MURI, grant\# FA9550-14-1-0389). Fabrication work was carried out in the Harvard Center for Nanoscale Systems, which is supported by the NSF. We thank Ms. Neha Singh from J. A. Woollam Company for ellipsometry analysis. We also thank Steven Byrnes and Pritpal Kanhaiya for reading the manuscript and their useful comments and $\mathrm{E}$. $\mathrm{Hu}$ for the supercontinuum laser (NKT "SuperK").

\section{REFERENCES}

(1) Pendry, J. B.; Schurig, D.; Smith, D. R. Science 2006, 312, 17801782

(2) Engheta, N.; Ziolkowski, R. W., Eds. Metamaterials: Physics and Engineering Explorations' Wiley-IEEE Press: Hoboken, NJ, 2006.

(3) Fang, N.; Lee, H.; Sun, C.; Zhang, X. Science 2005, 308, 534537.

(4) Schurig, D.; Mock, J. J.; Justice, B. J.; Cummer, S. A.; Pendry, J. B.; Starr, A. F.; Smith, D. R. Science 2006, 314, 977-980.

(5) Shelby, R. A.; Smith, D. R.; Shultz, S. Science 2001, 292, 77-79.

(6) Yu, N.; Capasso, F. Nat. Mater. 2014, 13, 139-150.

(7) Kildishev, A. V.; Boltasseva, A.; Shalaev, V. M. Science 2013, 339, 1232009.

(8) Yu, N.; Genevet, P.; Kats, M. A.; Aieta, F.; Tetienne, J. P.; Capasso, F.; Gaburro, Z. Science 2012, 334, 333-337.

(9) Larouche, S.; Tsai, Y.-J.; Tyler, T.; Jokerst, N. M.; Smith, D. R. Nat. Mater. 2012, 11, 450.

(10) Pinchuk, A. O.; Schatz, G. C. L. Opt. Soc. Am. A 2007, 24, A39A44.

(11) Kundtz, N.; Smith, D. R. Nat. Mater. 2010, 9, 129-132.

(12) Aieta, F.; Genevet, P.; Kats, M. A.; Yu, N.; Blanchard, R.; Gaburro, Z.; Capasso, F. Nano Lett. 2012, 12, 4932-4936.

(13) Khorasaninejad, M.; Aieta, F.; Kanhaiya, P.; Kats, M. A.; Genevet, P.; Rousso, D.; Capasso, F. Nano Lett. 2015, 15, 5358-5362.

(14) Monticone, F.; Estakhri, N. M.; Alù, A. Phys. Rev. Lett. 2013, 110, 203903.

(15) Walia, J.; Dhindsa, N.; Khorasaninejad, M.; Saini, S. S. Small 2014, 10, 144-151.

(16) Khorasaninejad, M.; Crozier, K. B. Nat. Commun. 2014, 5, 5386.

(17) Lin, D.; Fan, P.; Hasman, E.; Brongersma, M. L. Science 2014, 345, 298.

(18) Lin, J.; Mueller, J. P. B.; Wang, Q.; Yuan, G.; Antoniou, N.; Yuan, X.-C.; Capasso, F. Science 2013, 340, 331-334.

(19) Sun, S.; Yang, K.-Y.; Wang, C.-M.; Juan, T.-K.; Chen, W. T.; Liao, C. Y.; He, Q.; Xiao, S.; Kung, W.-T.; Guo, G.-Y.; Zhou, L.; Tsai, D. P. Nano Lett. 2012, 12, 6223-6229.

(20) Khorasaninejad, M.; Zhu, W.; Crozier, K. B. Optica 2015, 2, 376-382.

(21) Novotny, L.; Van Hulst, N. Nat. Photonics 2011, 5, 83-90.

(22) Zou, L.; Withayachumnankul, W.; Shah, C. M.; Mitchell, A.; Bhaskaran, M.; Sriram, S.; Fumeaux, C. Opt. Express 2013, 21, 13441352.

(23) Liu, N.; Liu, H.; Zhu, S. N.; Giessen, H. Nat. Photonics 2009, 3, $157-162$.

(24) Hutley, M. C. Diffraction Gratings; Academic Press: London, 1982.

(25) Stork, W.; Streibl, N.; Haidner, H.; Kipfer, P. Opt. Lett. 1991, 24, 1921-1923. 
(26) Lee, M. S. L.; Lalanne, P.; Rodier, J. C.; Chavel, P.; Cambril, E.; Chen, Y. I. Opt. A, Pure Appl. Opt. 2002, 4, 119-124.

(27) Fattal, D.; Li, J.; Peng, Z.; Fiorentino, M.; Beausoleil, R. G. Nat. Photonics 2010, 4, 466-470.

(28) Ribot, C.; Lee, M.; Collin, S.; Bansropun, S.; Plouhinec, P.; Thenot, D.; Cassette, S.; Loiseaux, B.; Lalanne, P. Adv. Opt. Mater. 2013, 1, 489-493.

(29) Buralli, D.; Morris, G. M. Appl. Opt. 1992, 31, 4389-4396.

(30) Hessler, T.; Rossi, M.; Kunz, R. E.; Gale, M. T. Appl. Opt. 1998, 37, 4069-4079.

(31) Hecht, E. Optics, 4th ed.; Addison-Wesley: Boston, MA, 2002. (32) Lalanne, P.; Astilean, S.; Chavel, P.; Cambril, E.; Launois, H. J. Opt. Soc. Am. A 1999, 16, 1143-1156.

(33) Khorasaninejad, M.; Abedzadeh, N.; Walia, J.; Patchett, S.; Saini, S. S. Nano Lett. 2012, 12, 4228-4234.

(34) Karagodsky, V.; Sedgwick, F. G.; Chang-Hasnain, C. J. Opt. Express 2010, 18, 16973-16988.

(35) Lewin, L. Proc. Inst. Elec. Eng. 1947, 94, 65-68. 